\title{
A New Bitterness Evaluation Index Obtained Using the Taste Sensor for 48 Active Pharmaceutical Ingredients of Pediatric Medicines
}

\author{
Honami Kojima, ${ }^{a}$ Toshio Kurihara, ${ }^{a}$ Miyako Yoshida, ${ }^{a}$ Tamami Haraguchi,${ }^{a}$ Haruka Nishikawa, ${ }^{a}$ \\ Saeri Ikegami, ${ }^{a}$ Takayoshi Okuno, ${ }^{a}$ Taku Yamashita, ${ }^{a}$ Junichi Nishikawa,${ }^{a}$ Hirofumi Tsujino, ${ }^{b}$ \\ Mitsuhiro Arisawa, ${ }^{b}$ Masaaki Habara, ${ }^{c}$ Hidekazu Ikezaki, ${ }^{c}$ and Takahiro Uchida*,a \\ ${ }^{a}$ Faculty of Pharmaceutical Science, Mukogawa Women's University; Nishinomiya, Hyogo 663-8179, Japan: \\ ${ }^{b}$ Graduate School of Pharmaceutical Sciences, Osaka University; 1-6 Yamadaoka, Suita, Osaka 565-0871, \\ Japan: and ${ }^{c}$ Intelligent Sensor Technology Inc.; Atsugi, Kanagawa 243-0032, Japan. \\ Received December 19, 2020; accepted March 11, 2021
}

The aim of this study was to evaluate bitterness by using "CCDP; Change in concentration-dependent potential" considering dose-dependency of active pharmaceutical ingredients (APIs) as new and useful bitterness evaluation index compared with bitter sensor output value which is conventional bitterness evaluation index for 48 pediatric medicines from the recent edition of the WHO model list of essential medicines for children (7th edn, 2019). Solutions $(0.01,0.03,0.1 \mathrm{mM})$ of the compounds were evaluated by an artificial taste sensor using membranes sensitive to bitterness. The dose-response slope of the sensor outputs was defined as CCDP. On the basis of principal component analysis of CCDPs, chlorpromazine hydrochloride, amitriptyline hydrochloride, propranolol hydrochloride, primaquine phosphate and haloperidol were predicted to express the strongest levels of basic bitterness, surpassing that of quinine hydrochloride. Correlation analysis (Fisher's exact tests and multiple regression analysis) was performed to determine the relation between CCDPs and various physicochemical properties participated in hydrophilicity and hydrophobicity. It is revealed that contribution physicochemical factors are different by individual basic bitterness sensor (AC0, ANO or BT0), and this result becomes the criterion of the sensor choice to evaluate basic bitterness intensity using basic bitterness sensors. Hydrophobic and hydrophilic interactions could be simulated by ligand docking modeling for haloperidol, miconazole and quinine hydrochloride. The pharmaceutical products need a bitterness evaluation in consideration of concentration-dependency to vary in a dose depending on a patient individual. Thus, it was concluded that CCDP correlated to hydrophilicity and hydrophobicity is useful as a bitterness evaluation index of APIs in pediatric medicines.

Key words taste sensor; bitterness; physicochemical property; pediatric medicine

\section{Introduction}

Drug formulations easily administrable to children are required for the efficacious and safe medication for children. ${ }^{1)}$ In general, drug formulations easily administrable to children have some attributes, for example, acceptable palatability, the ability to deliver precise drug doses, tablet size, etc. ${ }^{2-4)}$ Oral liquid dosage forms may be the most suitable dosage form for the treatment of pediatric patients, especially neonates. ${ }^{5)}$

Solubility, taste, stability and bioavailability are the major issues associated with the suitability of oral liquid dosage forms for children. ${ }^{6}$ The bitterness of oral pediatric medicines is often a big problem when bitter oral pediatric medicines need to be taken chronically and, in some cases, may even be life-threatening. ${ }^{7,8)}$

Different approaches have been used for taste-masking, including ion exchange, spheronization, encapsulation, granulation and the use of taste suppressants, sweeteners and flavors. ${ }^{9-14)}$ The bitterness suppression of active pharmaceutical ingredients with flavors and sweeteners which is the simplest and most frequently used approach is not always successful due to the large doses required and/or individual patient preferences.

Humans have 25 TASTE2 receptors (hTAS2R), a family of G-protein-coupled receptors for detecting bitterness. ${ }^{15)}$ Many bitter compounds activate multiple hTAS2Rs, while some bitter compounds are selective for a single hTAS2R. ${ }^{16)}$ The physi- cochemical properties (including solubility) of active pharmaceutical ingredients (APIs) are therefore likely to be important when considering the best way to mask their bitterness.

The commercially available artificial taste sensor system (Taste Sensing System, Incent Co., Ltd., Japan) developed by Toko with lipid/polymer membranes is widely and frequently used in pharmaceutical applications. ${ }^{17-23)}$ There are several types of bitterness or astringency sensors which have different components of lipids and plasticizers: $\mathrm{C} 00$ is sensitive to acidic bitter materials, such as diclofenac sodium which are non-steroidal anti-inflammatory drugs ${ }^{22)}$; and AE1 is sensitive to astringent materials such as tannic acid.; AC0 and AN0 are sensitive to basic materials, such as solifenacin succinate ${ }^{17)}$ or amlodipine besilate ${ }^{20)}$; BT0 is sensitive to hydrochloride salts, including quinine hydrochloride. ${ }^{18)}$ As bitterness taste sensors, taste sensors which detect various basic bitter substances ${ }^{26}$ ) and taste sensor which detect non-charged bitter substances ${ }^{23}$ ) have been development. The usefulness of the taste sensor in predicting the taste of various substances was proved by good correlations between results from the taste sensor and taste intensities evaluated by human gustatory sensation testing and taste sensors are now widely used application in the development of taste-masked formulations. The taste-masked formulation of cetirizine hydrochloride has been developed using a $\beta$-cyclodextrin and cherry/sucralose flavor system. ${ }^{24)}$ 
The taste-masking effect of carrageenan on donepezil hydrochloride was also demonstrated using the taste sensor, and a commercial product was developed. ${ }^{25}$ In a previous study, we investigated the correlation between bitterness sensor output values at a fixed dose of $0.1 \mathrm{mM}$ API more than that at $0.1 \mathrm{mM}$ quinine hydrochloride solution and typical physicochemical properties using Fisher's exact tests in order to try to classify bitter APIs according to these properties. ${ }^{26}$ In this paper, we targeted 48 APIs which were frequently and widely used for pediatric pharmaceuticals from newly updated WHO model list in 2019 (7th edn). Among APIs which used in the previous paper, ${ }^{26)} 19 \%$ of those (9 APIs) were exchanged. Secondly, the slope of semilogarithmic graph plotted with the abscissa denoting compound concentration (mM; log scale) and the ordinate denoting sensor output $(\mathrm{mV})$ was calculated and defined as "CCDP; Change in concentration-dependent potential." Moreover, CCDP was investigated to become new and useful bitterness evaluation index compared with bitter sensor output value which is conventional bitterness evaluation index for 48 bitter pediatric medicines from the recent edition of the WHO model list of essential medicines for children (7th edn, 2019).

In the present study, we selected $\log D$ value and number of hydrogen bond donor/acceptor and aromatic rings as parameters of physicochemical properties in addition to other properties performed in the previous study. ${ }^{26)}$

Forty-eight APIs were investigated correlations between CCDP and various physicochemical properties using Fisher's exact tests. Moreover, physicochemical properties which have high attribution to CCDP were extracted using multiple regression analysis, in order to verify the usefulness of CCDP as a bitterness evaluation index.

\section{Results and Discussion}

CCDPs of 48 APIs Using Five Bitterness Taste Sensors The 48 APIs used in the study are listed in Table 1. A semilogarithmic graph was plotted for each compound with abscissas denoting compound concentration (mM; log scale), and the ordinates denoting sensor output $(\mathrm{mV})$, as shown in Fig. 1. Several studies of electronic tongues for pharmaceutical analysis have been published. ${ }^{27,28)}$ The method for analyzing and evaluation bitterness intensity for APIs using a plural- ity of chemical sensors (or sensor arrays) was reported. The chemical diversity of the substances representing a bitter taste is huge and no universal chemical sensor (or sensor array) that would be able to provide the response towards all these diverse substances. While, sensor output value of bitter pharmaceutical drugs using individual chemical sensor should have correlation with the bitterness intensity of them, assuming pharmacological mechanism underlying bitterness response in organisms, which is, bitter substances bind to bitter receptor. Thus, we used 5 individual taste sensors (AC0, AN0 and BT0 for basic bitter substances and $\mathrm{C} 00$ and AE1 for acidic bitter substances) in this study. The slope of the plots was calculated and defined as CCDP. The CCDPs of the 48 APIs using five bitterness taste sensors are shown in Table 2. A concentration-dependent sensor output was confirmed with one of the bitterness taste sensors for 32 APIs (Acyclovir (ACV), Amitriptyline hydrochloride (AMT), Azathioprine (AZP), Azithromycin dehydrate (AZM), Chlorpromazine hydrochloride (CPZ), Clarithromycin (CTM), Digoxin (DGX), Doxycycline hyclate (DXC), Erythromycin (ETM), Ethosuximide (ESX), Fludrocortisone acetate (FCS), Furosemide (FUS), Haloperidol (HPD), Hydrochlorothiazide (HCT), Levofloxacin hemihydrate (LFX), Loratadine (LTD), Metformin hydrochloride (MEF), Metoclopramide hydrochloride (MCP), Metronidazole (MNZ), Miconazole (MCZ), Nitrofurantoin (NFT), Ondansetron hydrochloride dihydrate (ODS), Phenytoin (PHN), Primaquine phosphate (PRQ), Propranolol hydrochloride (PPL), Propylthiouracil (PTU), Quinine hydrochloride (QNN), Salbutamol sulfate (SBM), Spironolactone (SPL), Sulfamethoxazole (SMX), Trimethoprim (TMP) and Vancomycin hydrochloride (VCM)). No concentration-dependent sensor output was detected by any bitterness taste sensor with the other 16 compounds. Exactly, those 16 APIs have no report that bitterness of those APIs turned into clinical issue. CCDPs of 16 APIs (ACV, CTM, DGX, ETM, ESX, FCS, FUS, HCT, LFX, LTD, MEF, MCP, NFT, PTU, SBM and SPL) using C00 were much less than CCDP (-74.73) of iso-alpha-acid (acid bitterness standard substance for C00). Those APIs were evaluated slight bitterness intensity less than bitterness intensity of acidic bitterness standard substance. CCDPs of 6 APIs (AZP, ETM, FUS, MNZ, ODS and SMX) using AE1 were much less than CCDP (-10.48) of tannic acid (astringency standard substance

Table 1. Pediatric Pharmaceutical Medicines Used in This Study

\begin{tabular}{|c|c|c|}
\hline 1. Acetylsalicylic acid (ASA) & 17. Doxycycline hyclate (DXC) & 33. Metoclopramide hydrochloride (MCP) \\
\hline 2. Acyclovir (ACV) & 18. Erythromycin (ETM) & 34. Metronidazole (MNZ) \\
\hline 3. Allopurinol (ALP) & 19. Ethosuximide (ESX) & 35. Miconazole (MCZ) \\
\hline 4. Amitriptyline hydrochloride (AMT) & 20. Fluconazole (FCZ) & 36. Nitrofurantoin (NFT) \\
\hline 5. Amoxicillin trihydrate (AMX) & 21. Fludrocortisone acetate (FCS) & 37. Ondansetron hydrochloride dihydrate (ODS) \\
\hline 6. Azathioprine (AZP) & 22. Folic acid (FOL) & 38. Paracetamol (PCM) \\
\hline 7. Azithromycin dihydrate (AZM) & 23. Furosemide (FUS) & 39. Phenytoin (PHN) \\
\hline 8. Caffeine citrate (CAF) & 24. Haloperidol (HPD) & 40. Primaquine phosphate (PRQ) \\
\hline 9. Carbamazepine (CBZ) & 25. Hydrochlorothiazide (HCT) & 41. Propranolol hydrochloride (PPL) \\
\hline 10. Cefalexin (CFX) & 26. Ibuprofen (IBU) & 42. Propylthiouracil (PTU) \\
\hline 11. Chloramphenicol (CPH) & 27. Isoniazid (ISZ) & 43. Quinine hydrochloride (QNN) \\
\hline 12. Chlorpromazine hydrochloride (CPZ) & 28. Levofloxacin hemihydrate (LFX) & 44. Salbutamol sulfate (SBM) \\
\hline 13. Clarithromycin (CTM) & 29. Linezolid (LNZ) & 45. Spironolactone (SPL) \\
\hline 14. Dexamethasone (DMS) & 30. Loratadine (LTD) & 46. Sulfamethoxazole (SMX) \\
\hline 15. Diethylcarbamazine (DCZ) & 31. Metformin hydrochloride (MEF) & 47. Trimethoprim (TMP) \\
\hline 16. Digoxin (DGX) & 32. Methimazole (MMZ) & 48. Vancomycin hydrochloride (VCM) \\
\hline
\end{tabular}



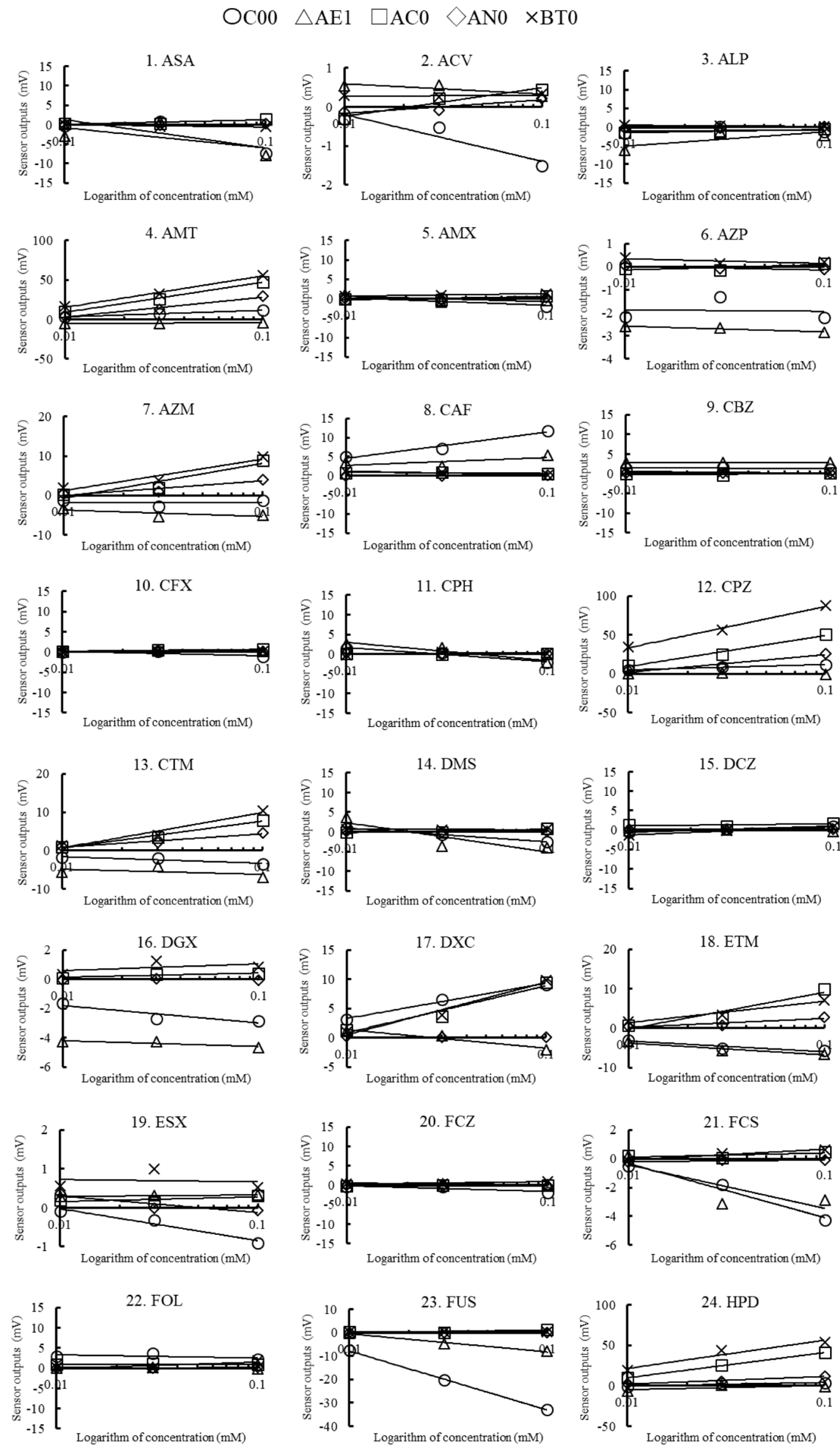

Fig. 1. Taste Sensor (C00 (R), AE1 (R), AC0 (cpa), AN0 (cpa) and BT0 (cpa)) Outputs (mV) in Response to 48 APIs

(R: relative sensor output; cpa: change in membrane potential caused by adsorption.) 

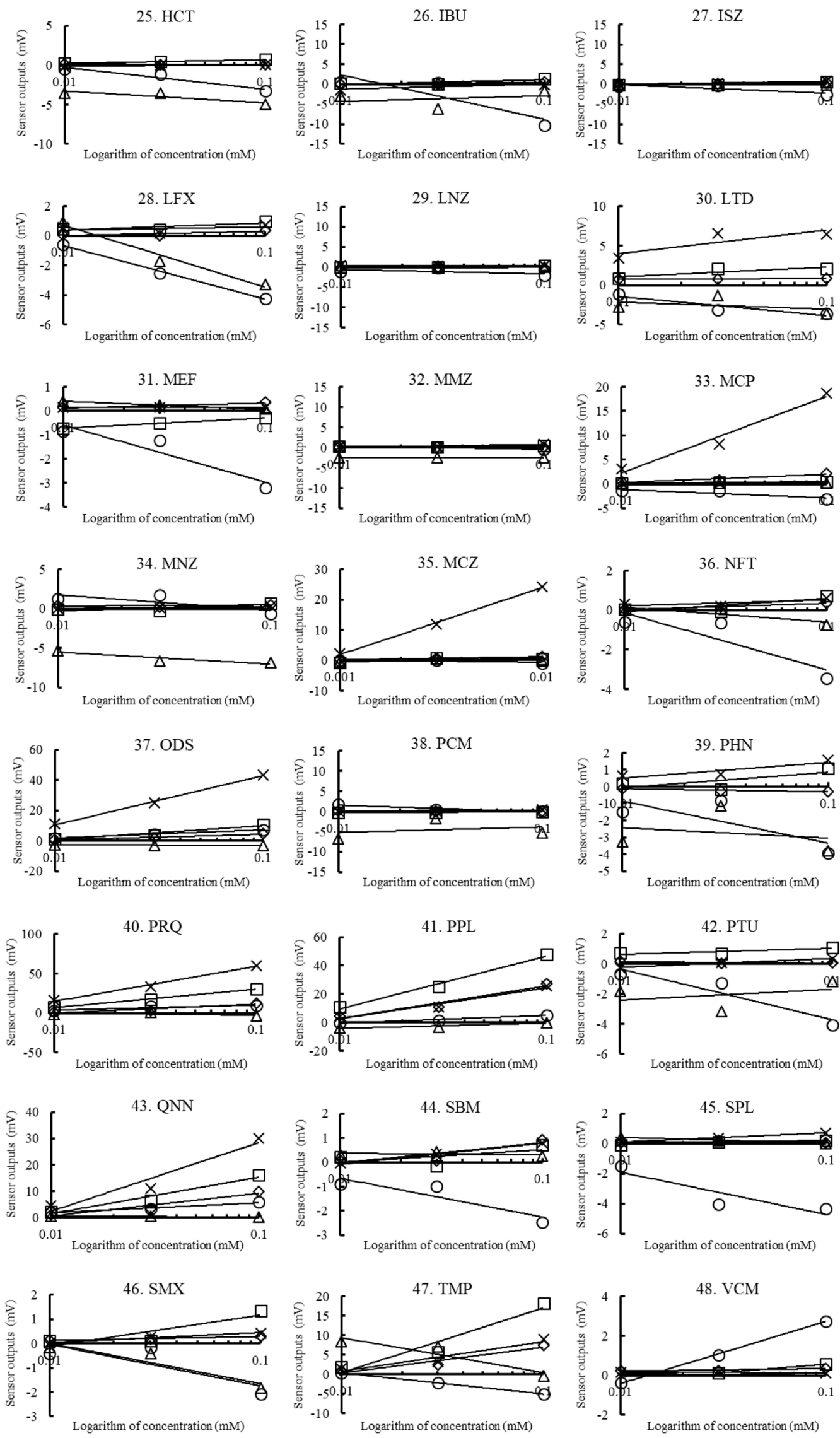

Fig. 1. Continued 
for AE1). Also, those APIs were evaluated slight bitterness intensity less than bitterness intensity of astringency standard substance. 18 APIs (Acetylsalicylic acid (ASA), ACV, Allopurinol (ALP), Amoxicillin trihydrate (AMX), AZP, Caffeine citrate (CAF), Carbamazepine (CBZ), Chloramphenicol (CPH), Diethylcarbamazine (DCZ), ESX, Fluconazole (FCZ), Folic acid (FOL), FUS, Ibuprofen (IBU), MEF, Paracetamol (PCM), PTU and SBM) were evaluated little bitter intensity because

Table 2. CCDPs (mV/log [mM]) from 5 Taste Sensor (C00 (R), AE1 (R), AC0 (cpa), AN0 (cpa) and BT0 (cpa)) Outputs in Response to 48 APIs

\begin{tabular}{|c|c|c|c|c|c|}
\hline $\begin{array}{c}\text { CCDP } \\
(\mathrm{mV} / \log [\mathrm{mM}])\end{array}$ & $\begin{array}{l}\mathrm{C} 00 \\
(\mathrm{R})\end{array}$ & $\begin{array}{l}\text { AE1 } \\
(\mathrm{R})\end{array}$ & $\begin{array}{l}\mathrm{AC} 0 \\
(\mathrm{cpa})\end{array}$ & $\begin{array}{l}\text { AN0 } \\
\text { (cpa) }\end{array}$ & $\begin{array}{l}\text { ВT0 } \\
\text { (сpa) }\end{array}$ \\
\hline ASA & 0 & 0 & 0 & 0 & 0 \\
\hline $\mathrm{ACV}$ & -0.53 & 0 & 0 & 0 & 0 \\
\hline ALP & 0 & 0 & 0 & 0 & 0 \\
\hline AMT & 0 & 0 & 16.57 & 10.86 & 17.26 \\
\hline AMX & 0 & 0 & 0 & 0 & 0 \\
\hline AZP & 0 & -0.11 & 0 & 0 & 0 \\
\hline AZM & 0 & 0 & 3.81 & 1.61 & 3.47 \\
\hline CAF & 0 & 0 & 0 & 0 & 0 \\
\hline CBZ & 0 & 0 & 0 & 0 & 0 \\
\hline CFX & 0 & 0 & 0 & 0 & 0 \\
\hline $\mathrm{CPH}$ & 0 & 0 & 0 & 0 & 0 \\
\hline $\mathrm{CPZ}$ & 0 & 0 & 17.28 & 9.84 & 23.03 \\
\hline CTM & -0.74 & 0 & 3.04 & 1.5 & 3.98 \\
\hline DMS & 0 & 0 & 0 & 0 & 0 \\
\hline $\mathrm{DCZ}$ & 0 & 0 & 0 & 0 & 0 \\
\hline DGX & -0.52 & 0 & 0.13 & 0 & 0 \\
\hline $\mathrm{DXC}$ & 0 & 0 & 3.5 & 0 & 3.9 \\
\hline ETM & -1.19 & -1.32 & 4.04 & 0.96 & 2.36 \\
\hline ESX & -0.36 & 0 & 0 & 0 & 0 \\
\hline FCZ & 0 & 0 & 0 & 0 & 0 \\
\hline FCS & -1.63 & 0 & 0 & 0 & 0 \\
\hline FOL & 0 & 0 & 0 & 0 & 0 \\
\hline FUS & -11 & -3.36 & 0 & 0 & 0 \\
\hline HPD & 0 & 0 & 13.49 & 4.11 & 15.14 \\
\hline HCT & -1.2 & 0 & 0.21 & 0 & 0 \\
\hline IBU & 0 & 0 & 0 & 0 & 0 \\
\hline ISZ & 0 & 0 & 0 & 0 & 0 \\
\hline LFX & -1.58 & 0 & 0 & 0 & 0 \\
\hline LNZ & 0 & 0 & 0 & 0 & 0 \\
\hline LTD & -1.07 & 0 & 0.53 & 0.03 & 0 \\
\hline MEF & -1.04 & 0 & 0 & 0 & 0 \\
\hline MMZ & 0 & 0 & 0 & 0 & 0 \\
\hline $\mathrm{MCP}$ & -0.74 & 0 & 0.18 & 0.73 & 6.82 \\
\hline MNZ & 0 & -0.67 & 0 & 0 & 0.1 \\
\hline $\mathrm{MCZ}$ & 0 & 0 & 0 & 0.43 & 9.56 \\
\hline NFT & -1.26 & 0 & 0.3 & 0 & 0 \\
\hline ODS & 0 & -0.28 & 3.94 & 1.62 & 14.06 \\
\hline PCM & 0 & 0 & 0 & 0 & 0 \\
\hline PHN & 0 & 0 & 0 & 0 & 0.4 \\
\hline PRQ & 0 & 0 & 10.2 & 4.87 & 19.17 \\
\hline PPL & 0 & 0 & 16.11 & 9.92 & 12.29 \\
\hline PTU & -1.47 & 0 & 0 & 0 & 0 \\
\hline QNN & 0 & 0 & 6 & 3.77 & 11.3 \\
\hline SBM & -0.7 & 0 & 0 & 0 & 0 \\
\hline SPL & -1.23 & 0 & 0 & 0 & 0.27 \\
\hline SMX & 0 & -0.73 & 0 & 0.07 & 0.18 \\
\hline TMP & 0 & 0 & 7.23 & 2.85 & 3.28 \\
\hline VCM & 0 & 0 & 0.37 & 0.06 & 0 \\
\hline
\end{tabular}

(R: Relative sensor output; cpa: Change in membrane potential caused by adsorption) of little sensor output value at $0.1 \mathrm{mM}$ API in compared with that at $0.1 \mathrm{mM}$ quinine hydrochloride in our previous study. They showed no concentration-dependent responses to basic bitterness sensors, namely, CCDPs of them were not obtained in this study, consequently, they were predicted to show little bitterness intensity even in a high dose. On the other hand, 6 APIs (DGX, MNZ, NFT, PHN, SPL and VCM) evaluated little bitter intensity in our previous study were confirmed to show concentration-dependent responses to basic bitterness sensors in this study. So, they were predicted to show potent bitterness intensity at a high dose. Consequently, it was suggested that CCDP considering dose-dependency (i.e., slope) of API was able to show degree of the bitterness of those APIs for an inherent value of API as a bitterness evaluation index better than bitter sensor output value which is conventional bitterness evaluation index.

The conventional taste sensor cannot measure non-charged bitter substance such as caffeine. However, caffeine which is one of non-charged bitter substances was reported to be ligand of hTAS2Rs. ${ }^{15)}$ In this study, the lipid/polymer membranes used as bitterness taste sensors. Electrostatic interaction and hydrophobic interaction might be participated in the adsorption of APIs to the lipid/polymer membranes used as bitterness taste sensors. Concentration-dependent increase of hydrophobic interaction between non-charged substances (DXC and ESX) and the lipid/polymer membranes was suggested to show CCDP value in this study.

Tastes of 48 APIs Predicted by PCA of CCDPs The taste maps for each API, based on PCA using the five CCDPs, are shown in Fig. 2. PCA was used to estimate the largest and second-largest relative contributing factors ( $\mathrm{PC} 1$ and $\mathrm{PC} 2$, respectively). The relative contributions of $\mathrm{PC} 1$ and $\mathrm{PC} 2$ were 56.1 and $35.3 \%$, respectively. Using PCA, substances with a large main component load can be identified. The principal component loadings of the five bitterness taste sensors were described as follows: AN0: PC1 0.96, PC2 0.20; AC0: PC1 0.94, PC2 0.20; BT0: PC1 0.91, PC2 0.20; AE1: PC1 0.27, PC2 -0.92; and $\mathrm{C} 00$ : $\mathrm{PC} 10.34, \mathrm{PC} 2-0.90$. Consequently, the CCDPs of AN0, AC0 and BT0 (for basic bitterness) contributed to $\mathrm{PC} 1$ rather than $\mathrm{PC} 2$, while those of $\mathrm{AE} 1$ and $\mathrm{C} 00$ (for astringency or acidic bitterness, respectively) contributed to PC2 rather than PC1. In Fig. 2, the direction of the PC1 axis

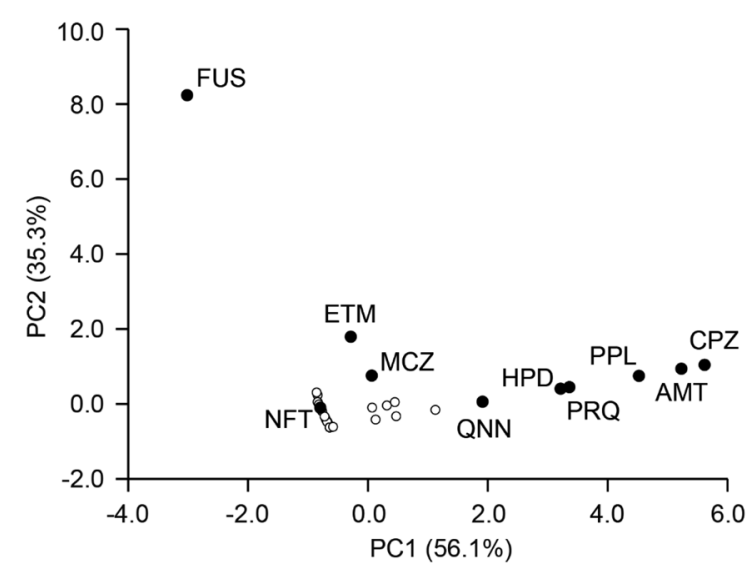

Fig. 2. PCA of CCDPs (C00, AE1, AC0, AN0 and BT0) in Response to 48 APIs

The remarkable data were represented as filled circles with the name of APIs, and the others are shown in open circles, respectively. 
Table 3. Physicochemical Properties of 48 APIs (Data from DRUGBANK or Marvinsketch)

\begin{tabular}{|c|c|c|c|c|c|c|c|c|c|c|}
\hline Compounds & MW & $\log P$ & $\log D$ & $\log S$ & Charge & PSA & Polarizability & $\begin{array}{l}\text { Hydrogen } \\
\text { bond donor }\end{array}$ & $\begin{array}{c}\text { Hydrogen } \\
\text { bond acceptor }\end{array}$ & $\begin{array}{c}\text { Aromatic } \\
\text { rings }\end{array}$ \\
\hline ASA & 180 & 1.43 & -2.29 & -2.1 & -1 & 63.6 & 17.1 & 1 & 2 & 1 \\
\hline $\mathrm{ACV}$ & 225 & -0.95 & -1.54 & -1.4 & 0 & 114.8 & 21.5 & 3 & 4 & 2 \\
\hline ALP & 136 & -1.7 & -0.73 & -1.4 & 0 & 65.9 & 11.7 & 2 & 3 & 2 \\
\hline AMT & 277 & 5.1 & 1.96 & -4.8 & 1 & 3.24 & 33.7 & 0 & 1 & 3 \\
\hline AMX & 365 & 0.75 & -1.01 & -2.6 & 0 & 133 & 35.5 & 3 & 2 & 3 \\
\hline AZP & 277 & 0.84 & 1.21 & -2.4 & 0 & 118.1 & 24.3 & 1 & 6 & 3 \\
\hline AZM & 749 & 3.03 & -2.87 & -3.2 & 2 & 180.1 & 83.1 & 5 & 2 & 3 \\
\hline CAF & 194 & -0.24 & -9.8 & -1.2 & 0 & 58.4 & 19 & 0 & 6 & 2 \\
\hline $\mathrm{CBZ}$ & 236 & 2.1 & 2.77 & -3.2 & 0 & 46.3 & 25 & 1 & 2 & 3 \\
\hline CFX & 347 & 0.55 & -2.23 & -3.1 & 0 & 112.7 & 32.5 & 3 & 5 & 3 \\
\hline $\mathrm{CPH}$ & 323 & 1.15 & 0.82 & -2.8 & 0 & 115.4 & 28.1 & 3 & 2 & 1 \\
\hline $\mathrm{CPZ}$ & 319 & 5.18 & 0.97 & -4.9 & 1 & 6.5 & 35.1 & 0 & 2 & 3 \\
\hline CTM & 255 & 3.44 & 1.65 & -3.5 & 1 & 12.5 & 29.9 & 0 & 1 & 2 \\
\hline DMS & 392 & 1.68 & 1.68 & -3.9 & 0 & 94.8 & 40.7 & 3 & 5 & 4 \\
\hline $\mathrm{DCZ}$ & 199 & 0.9 & -0.26 & 0.07 & 0 & 26.8 & 22.9 & 0 & 4 & 1 \\
\hline DGX & 780 & 1.04 & 2.21 & -3.8 & 0 & 203.1 & 84.8 & 6 & 1 & 8 \\
\hline DXC & 444 & -0.72 & 0.76 & -2.8 & 0 & 181.6 & 43.7 & 6 & 4 & 4 \\
\hline ETM & 734 & 2.37 & 1.29 & -3.2 & 1 & 193.9 & 78.2 & 5 & 3 & 3 \\
\hline ESX & 141 & 0.1 & 0.55 & -0.15 & 0 & 46.2 & 14.5 & 1 & 2 & 1 \\
\hline $\mathrm{FCZ}$ & 306 & 0.58 & 0.12 & -2.3 & 0 & 81.7 & 26.5 & 1 & 8 & 3 \\
\hline FCS & 380 & 1.32 & 1.76 & -3.2 & 0 & 94.8 & 39.5 & 3 & 5 & 4 \\
\hline FOL & 441 & -0.04 & -7.17 & -3.8 & -2 & 209 & 42.1 & 6 & 7 & 3 \\
\hline FUS & 330 & 2.71 & -0.76 & -3.4 & -1 & 122.6 & 30.6 & 3 & 3 & 2 \\
\hline HPD & 375 & 3.7 & 2.39 & -4.9 & 1 & 40.5 & 39.2 & 1 & 3 & 3 \\
\hline HCT & 297 & -0.16 & -0.58 & -2.1 & 0 & 118.4 & 25.4 & 3 & 4 & 2 \\
\hline IBU & 206 & 3.5 & 1.9 & -3.5 & -1 & 37.3 & 23.8 & 1 & 1 & 1 \\
\hline ISZ & 137 & -0.71 & -0.69 & -0.59 & 0 & 68 & 13.2 & 2 & 2 & 1 \\
\hline LFX & 361 & 1.32 & -0.65 & -2.4 & -1 & 73.3 & 36.7 & 1 & 7 & 4 \\
\hline LNZ & 337 & 0.64 & -0.48 & -2.4 & 0 & 71.1 & 34.1 & 1 & 5 & 3 \\
\hline LTD & 382 & 4.8 & 4.55 & -4.5 & 0 & 42.4 & 41.7 & 0 & 3 & 4 \\
\hline MEF & 129 & -1.8 & -7.08 & -2 & 2 & 89 & 13.4 & 4 & 1 & 0 \\
\hline MMZ & 114 & 0 & -1.96 & -1 & 0 & 15.3 & 11.6 & 1 & 0 & 1 \\
\hline MCP & 299 & 2.18 & -0.83 & -3 & 1 & 67.6 & 32.7 & 2 & 2 & 1 \\
\hline MNZ & 171 & -0.15 & -0.46 & -1.5 & 0 & 83.9 & 15.8 & 1 & 3 & 1 \\
\hline $\mathrm{MCZ}$ & 416 & 5.96 & 6.7 & -5.7 & 0 & 27.1 & 39.5 & 0 & 2 & 3 \\
\hline NFT & 238 & 0.03 & -1.93 & -2.8 & 0 & 118.1 & 20.5 & 1 & 5 & 2 \\
\hline ODS & 293 & 2.56 & 2.79 & -3.1 & 1 & 39.8 & 33.2 & 0 & 4 & 4 \\
\hline PCM & 151 & 0.51 & 0.91 & -1.6 & 0 & 49.3 & 15.5 & 2 & 1 & 1 \\
\hline PHN & 252 & 2.26 & 2.15 & -3.6 & 0 & 58.2 & 25.5 & 2 & 2 & 3 \\
\hline PRQ & 259 & 2.76 & 1.27 & -3.7 & 1 & 60.2 & 29.9 & 2 & 1 & 2 \\
\hline PPL & 259 & 3.03 & -0.13 & -3.5 & 1 & 41.5 & 30 & 2 & 0 & 2 \\
\hline PTU & 170 & 1.53 & 1.9 & -2.6 & 0 & 41.1 & 17.8 & 2 & 1 & 1 \\
\hline QNN & 324 & 2.82 & 0.29 & $-\mathbf{3}$ & 1 & 45.6 & 36 & 1 & 2 & 3 \\
\hline SBM & 239 & 0.44 & -1.76 & -2 & 1 & 72.7 & 26.9 & 4 & 0 & 1 \\
\hline SPL & 416 & 3.1 & 3.64 & -5.3 & 0 & 60.4 & 46 & 0 & 3 & 5 \\
\hline SMX & 253 & 0.79 & 0.24 & -2.7 & -1 & 98.2 & 25 & 2 & 3 & 2 \\
\hline TMP & 290 & 0.91 & 0.8 & 1.28 & 1 & 105.5 & 29.7 & 2 & 7 & 2 \\
\hline VCM & 1449 & 1.11 & -5.42 & -3.8 & 1 & 530.5 & 138.7 & 19 & 7 & 7 \\
\hline
\end{tabular}

reflects the contribution of basic bitterness and the direction of the PC2 axis reflects the contribution of astringency or acidic bitterness. On the basis of the responses to the taste sensor, CPZ, AMT, PPL, PRQ and HPD were predicted to express strong basic bitterness, surpassing that of QNN, which was used as the standard of bitterness. MCZ, NFT and ETM were also predicted to be basic bitter compounds. FUS was predicted to show astringency and acidic bitterness, based on the responses of both AE1 and $\mathrm{C} 00$.
Physicochemical Properties of 48 APIs The following physicochemical properties of the 48 APIs were extracted from the DRUGBANK database: molecular weight (MW), $\log P$, $\log S$ (predicted by ALOGPS), physiological charge, PSA and polarizability. $\log D$ was obtained by Marvinsketch. These are summarized in Table 3. The values in bold are either equal to or greater than the values of QNN for MW (324), the $\log P$ value (2.82), the $\log D$ value (0.29), the $\log S$ value $(-3)$, the physiological charge $(|1|)$, the PSA $\left(45.6 \AA^{2}\right)$, the polarizabil- 
Table 4. Correlation Coefficients by Fisher's Exact Test between Physicochemical Properties and CCDP

\begin{tabular}{|c|c|c|c|c|c|c|c|c|c|c|}
\hline & $\begin{array}{l}\text { MW } \\
\geq 324\end{array}$ & $\begin{array}{l}\log P \\
\geq 2.82\end{array}$ & $\begin{array}{l}\log D \\
\geq 0.29\end{array}$ & $\begin{array}{l}\log S \\
<-3\end{array}$ & $\begin{array}{c}\text { Charge } \\
\geq|1|\end{array}$ & $\begin{array}{c}\text { PSA } \\
<45.59 \AA^{2}\end{array}$ & $\begin{array}{l}\text { Polarizability } \\
\geq 35.96 \AA^{3}\end{array}$ & $\begin{array}{l}\text { Hydrogen } \\
\text { bond } \\
\text { donor } \\
\geq 1\end{array}$ & $\begin{array}{l}\text { Hydrogen } \\
\text { bond } \\
\text { acceptor } \\
\geq 2\end{array}$ & $\begin{array}{c}\text { Aromatic } \\
\text { rings } \\
\geq 3\end{array}$ \\
\hline $\mathrm{AC} 0$ & 0.08 & $0.48^{a)}$ & 0.21 & 0.3 & $0.47^{a)}$ & 0.31 & 0.01 & 0.1 & 0.2 & 0.06 \\
\hline AN0 & 0.03 & $0.54^{a)}$ & 0.28 & $0.38^{b)}$ & $0.43^{a)}$ & $0.36^{a)}$ & 0.03 & 0.14 & 0.24 & 0.13 \\
\hline BT0 & 0.08 & $0.48^{a)}$ & $0.33^{b)}$ & $0.41^{a)}$ & $0.41^{a)}$ & $0.44^{a)}$ & 0.12 & $0.41^{b)}$ & 0.06 & 0.3 \\
\hline $\mathrm{C} 00$ & 0.09 & 0.07 & 0.03 & 0 & 0 & 0.1 & 0.13 & 0 & 0.14 & 0.18 \\
\hline $\mathrm{AE} 1$ & 0.05 & 0.08 & 0.09 & 0.06 & 0.11 & 0.03 & 0.01 & 0.1 & 0.23 & 0.06 \\
\hline
\end{tabular}

a) $p<0.01$, Fisher's exact test. b) $p<0.05$, Fisher's exact test.

ity $\left(36.0 \AA^{3}\right)$, the hydrogen bond donor value (1) and acceptor value (2), and the number of aromatic rings (3), respectively.

Correlation between CCDPs and Physicochemical Properties Fisher's exact tests were carried out to examine the correlations between the physicochemical properties participated in an electric charge and hydrophobicity and CCDPs (Table 4). $\log P$ values $\geq 2.82$ and absolute values of physiological charge $\geq|1|$ significantly correlated with the responses of AN0, AC0 and BT0, respectively $(p<0.01)$. Log $S$ values $<-3$ significantly correlated with the responses of BT0 $(p<0.01)$ and AN0 $(p<0.05)$. PSA $<45.59 \AA^{2}$ significantly correlated with the responses of AN0 and BT0 $(p<0.01)$. There were no significant correlations between polarizability or hydrogen bond acceptor or aromatic rings and the responses of the taste sensor. Judging from the fact that high $\log P$ values $(\geq 2.82)$ absolute values of physiological charge $(\geq|1|)$, low $\log S$ values $(<-3)$ and small PSA $\left(<45.59 \AA^{2}\right)$ significantly correlated with the responses of basic bitterness sensors, it can be concluded that high lipophilicity, high physiological charge, low solubility and small PSA are factors associated with CCDP of basic bitterness. Bitter compounds are absorbed on the hydrophobic part of the taste sensor membrane and cause a change in membrane potential by changing the charge density. ${ }^{19)}$ Roland et al. suggested that hydrophobic interaction is important for binding to bitter taste receptor hTAS2R14 which is broadly tuned by a large number of agonists. ${ }^{29,30)}$ These references support our finding of a relation between bitterness and high lipophilicity (low solubility), physiological charge. PSA, defined as the sum of the surfaces of polar atoms in a molecule, is known to be a parameter predictive of intestinal absorption. ${ }^{31)}$ Our results suggested that a low PSA value $\left(<45.59 \AA^{2}\right)$ may be linked with bitterness, although there have been no previous reports of a relationship between bitterness and PSA. Low PSA might interact with hydrophobicity of APIs. Hydrophobicity of APIs was suggested to have effect on affinity of APIs to bitterness receptor. Clark ${ }^{32)}$ suggested that a poorly absorbed molecule will have PSA $>140 \AA^{2}$. In our study, 6 of the 48 APIs had a PSA $>140 \AA^{2}$. Molar refractivity is related to dispersive interaction which is one of three most important forces (hydrophobic, dispersive and electrostatic interactions) in biochemical ligand binding, and the molecular orbital charge distribution or the electrostatic potential at the van der Waals radius may be used for modeling the electrostatic interaction, ${ }^{33)}$ however, there was no significant correlation between refractivity and sensor output value at $0.1 \mathrm{mM}$ of bitter taste sensor in our previous study. ${ }^{26)}$ So, refractivity was estimated to have little attribution to bitterness intensity was omitted in analysis of this study.
There are significant correlations between $\log P$ and CCDPs in basic bitterness sensors namely, AC0, AN0 and BT0 ( $p<0.01$, respectively), Charge and CCDPs in basic bitterness sensors $(p<0.01$, respectively). These significant correlations were the same as the correlation between $\log P$ and basic bitterness sensors outputs at $0.1 \mathrm{mM}$ APIs in the previous study. In the present study, in related to AN0, there was significant correlation between $\log S$ and CCDP $(p<0.05)$, whereas no significant correlation between $\log S$ and sensor output at $0.1 \mathrm{mM}$ APIs. $\log S$ was likely to show hydrophilicity of APIs related in adsorption of APIs to AN0 membrane. Especially in BT0, there are significant correlations between $\log D$ and CCDP, hydrogen bond donor and CCDP $(p<0.05$, respectively). $\log D$ was suggested to show hydrophobicity of APIs related in adsorption of APIs to basic bitterness sensor membrane and the number of hydrogen bond donor is suggested to show hydrophilicity of APIs related in adsorption of API to basic bitterness sensor membrane. CCDPs would have higher correlation with physicochemical parameters of API hydrophobicity or hydrophilicity related in adsorption of APIs to basic bitterness sensor membranes compared to sensor outputs at $0.1 \mathrm{mM}$ APIs.

In this method, affinities of APIs to basic bitterness sensors were confirmed using concentration-dependent responses of APIs to basic bitterness sensors, namely, CCDPs. And affinities of APIs to basic bitterness sensors would predict affinities of APIs to bitterness receptor in human body. Using CCDPs, comparison in affinities which have effect on bitterness intensity of various APIs would be possible and characterization of APIs at the same level of affinity would lead to the development of the bitterness masking method that was common to those APIs. Multiple regression analysis was performed to investigate contribution of physicochemical properties (explanatory variables) to CCDPs of AC0, AN0 and BT0 (target variables) and partial regression coefficients were obtained respectively. As the results, the multiple regression formula for AC0 (1) $(R=0.48, p<0.05)$ described below was obtained.

$$
\text { Predicted CCDP }=1.62 \log P+1.88
$$

$\log P$ was found to have significant partial regression coefficient in $\mathrm{ACO}(p<0.05)$. Therefore, we may conclude that especially $\log P$ is correlated with basic bitterness evaluated using ACO.

The multiple regression formula for AN0 (2) $(R=0.57$, $p<0.05)$ described below was obtained.

$$
\text { Predicted CCDP }=1.59 \log P-1.15
$$



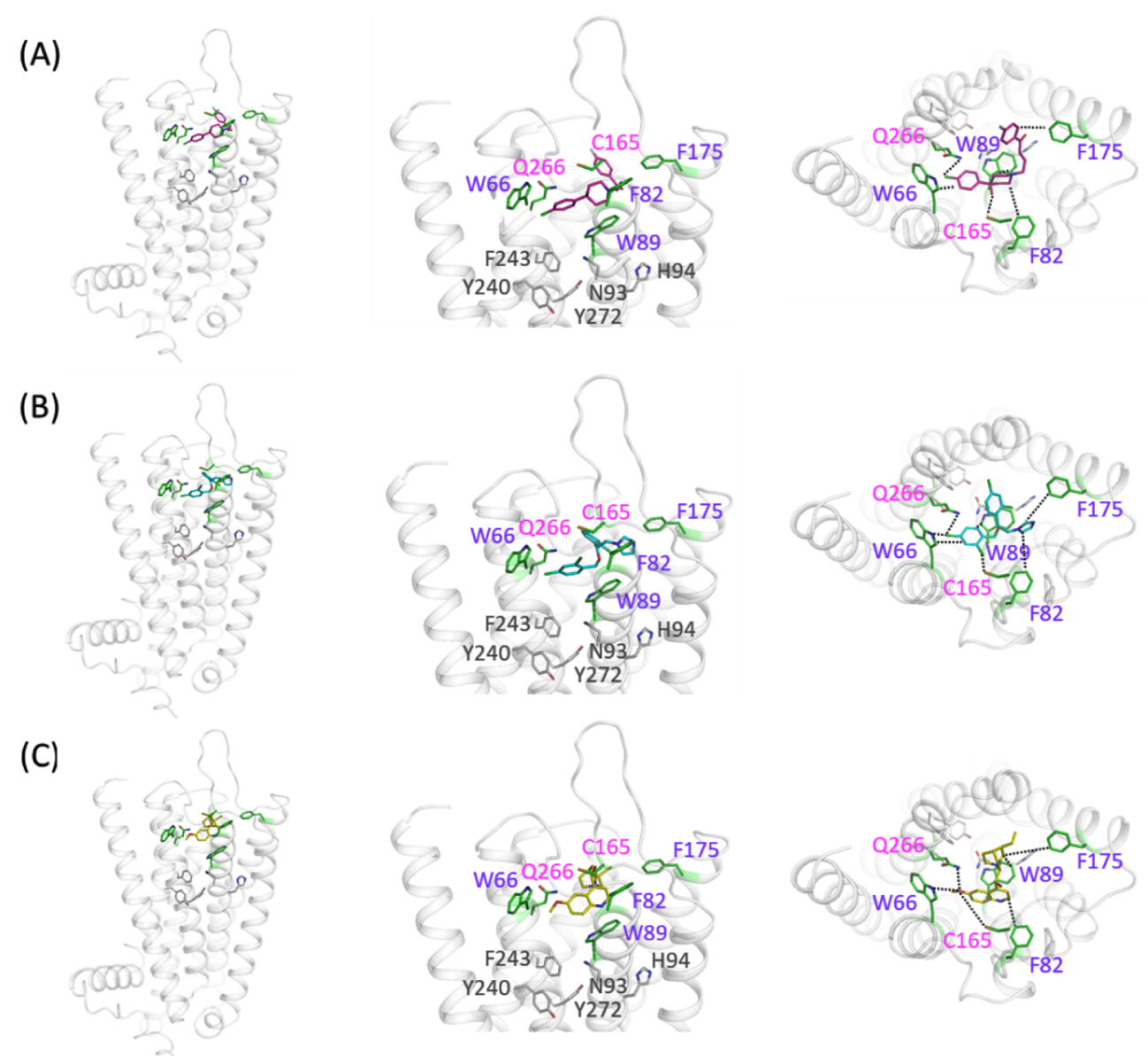

Fig. 3. Docking Simulations of Haloperidol (A), Miconazole (B), and Quinine Hydrochloride (C) into the Constructed hTAS2R14 Model

The simulated structures are represented in a ribbon model in entirely (left panels) and partially (middle and right panels). The docked ligands, HPD, QNN and MCZ are shown as stick models in magenta, cyan and yellow, respectively, for carbon; nitrogen and oxygen are colored in blue and red, respectively. Several nearby residues are also represented as stick models in green for carbon, and the dashed lines shown solely in right panels indicate possible interactions between ligands and the amino acids labeled for electrostatic (magenta) and hydrophobic (purple) interactions. The tagged amino acids suggested previously for the orthostatic site are also shown in left panels as stick models in gray for carbons. The structures are shown as viewed from the membrane (left and middle) and extracellular space (right). (Color figure can be accessed in the online version.)

$\log P$ was found to have significant partial regression coefficient in AN0 $(p<0.05)$. Therefore, we may conclude that $\log P$ is correlated with basic bitterness evaluated using ANO. There was no difference in significant partial regression coefficient between $\mathrm{AC} 0$ and $\mathrm{ANO}$ in this study.

The multiple regression formula for BT0 (3) $(R=0.69$, $p<0.01)$ described below was obtained.

$$
\text { Predicted CCDP }=6.23 \text { Charge }-0.07 \text { PSA }+8.70
$$

Charge $(p<0.01)$ and PSA $(p<0.01)$ were found to be partial regression coefficients in BT0. Therefore, we may conclude that especially Charge and PSA are correlated with basic bitterness evaluated using BT0. No universal chemical sensor (or sensor array) that would be able to provide the response towards all these diverse substances. However, at least, bitterness sensors in this study were reported that sensor output value of bitter pharmaceutical drugs using individual chemical sensor showed correlation with the bitterness intensity of those drugs by gustatory sensation test. It is revealed that contribution physicochemical factors are different by sensor, and this result becomes the criterion of the sensor choice. The pharmaceutical products need a bitterness evaluation in consideration of concentration-dependency to vary in a dose depending on a patient individual. Thus, the proposed index would have practical utility.

Prediction of Haloperidol, Miconazole and Quinine Hydrochloride Binding to hTAS2R14 Using a Docking Model hTAS2R14 was chosen because responses of basic bitterness sensors were found to be correlated with responses of hTAS2R14 in our previous study. ${ }^{34)}$ It was reported that HPD, MCZ and QNN are typical ligands to hTAS2R14. ${ }^{15,35)}$ The prediction of HPD, MCZ, QNN binding to hTAS2R14 using docking model was to be helpful concluding the mechanism underlying obtainment of CCDPs using basic bitterness sensors. The predicted model structure of hTAS2R14 was built and docking simulations performed for HPD, MCZ and QNN (Fig. 3). The nearest amino acids (within $5 \AA$ ) and the amino acids previously suggested to interact with the simulated ligands ${ }^{36,37)}$ are depicted as stick models, and distances to the docked compounds are shown for several residues as dashed lines. In Fig. 3A, the structure of HPD docked to the hTAS2R14 model shows that HPD is $4.6 \AA$ from Q266. This residue was also found to be located near to the docking positions of MCZ and QNN, with estimated distances of $3.9 \AA$ and $3.7 \AA$, respectively. HPD also docked relatively close to

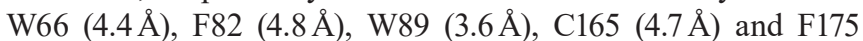

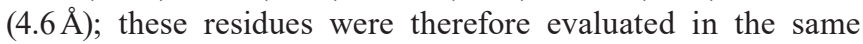
manner for MCZ and QNN (Figs. 3B and C, respectively). It is 
interesting that some of these residues are conserved in other subfamilies of hTAS2Rs. It has been reported that a model structure of hTAS2R10 showed decreases of substrate binding with computational mutations at the residues (W88, N92, Q93, Y239 and F266) designated as the sites of interaction between hTAS2R10 and its substrates. ${ }^{29)}$ Our simulated binding site was also in good agreement with a vestibular binding site designated on a constructed structure of hTAS2R46. ${ }^{38)}$ The vestibular binding site in hTAS2R46 was formed by W66 and E265, corresponding to W66 and Q266 in hTAS2R14, and these residues were also constructed as a binding pocket in our simulated structures, with distances to the docked compounds of 4.0-4.4 $\AA$ for W66 and 3.7-4.6 $\AA$ for Q266. Further simulations for the constructed hTASR46 structure revealed another binding site, an orthosteric one, constructed by residues H93, Y241, F242 and F269. Although the corresponding residues in our hTAS2R14 model, H94, Y240, F243 and Y272, were located deeper inside and further from the docked substrate, further simulations using our model may reveal a deeper binding site, as discussed in previous studies. ${ }^{16,36,39)}$ Consequently, our docking simulations for the constructed hTAS2R14 model appear to be reliable, and these results suggest that the studied compounds interact with hTAS2R14 with both hydrophobic and electrostatic interactions, as reported previously.

\section{Conclusion}

The aim of this study was to evaluate bitterness by using "CCDP; Change in concentration-dependent potential" was useful bitterness evaluation index compared with bitter sensor output value which is conventional bitterness evaluation index for 48 bitter pediatric medicines from the recent edition of the WHO model list of essential medicines for children (7th edn, 2019). Parameters which participated in an electric charge and hydrophobicity and performed a correlation analysis using Fischer's exact test against "CCDP" assuming as a bitterness evaluation index. Moreover, multiple regression formula deriving predicted CCDP for basic bitterness using AC0, AN0 and BT0 were obtained by multiple regression test. It is revealed that contribution physicochemical factors are different by individual sensor, and this result becomes the criterion of the sensor choice. The contribution of these physiological parameters of API in bitterness response in human was supported by the result from docking simulation. The pharmaceutical products need a bitterness evaluation in consideration of concentrationdependency to vary in a dose depending on a patient individual. "CCDP" would be useful for comparison of bitterness intensity of each APIs and prediction of bitterness intensity of APIs in individual dose with wide range.

\section{Experimental}

Materials Acetylsalicylic acid (ASA), Allopurinol (ALP), Amitriptyline hydrochloride (AMT), Azathioprine (AZP), Caffeine citrate (CAF), Carbamazepine (CBZ), Cefalexin (CFX), Chloramphenicol (CPH), Chlorpromazine hydrochloride (CPZ), Dexamethasone (DMS), Diethylcarbamazine (DCZ), Erythromycin (ETM), Fludrocortisone acetate (FCS), Folic acid (FOL), Haloperidol (HPD), Hydrochlorothiazide (HCT), Ibuprofen (IBU), Levofloxacin hemihydrate (LFX), Linezolid (LNZ), Metronidazole (MNZ), Phenytoin (PHN), Salbutamol sulfate (SBM), Spironolactone (SPL), Sulfamethoxazole (SMX), Trimethoprim (TMP) and Vancomycin hydrochlo- ride (VCM) were purchased from FUJIFILM Wako Pure Chemical Corporation (Japan), Acyclovir (ACV), Methimazole (MMZ), Primaquine phosphate (PRQ) from Combi-Blocks Inc. (U.S.A.), Amoxicillin trihydrate (AMX), Azithromycin dihydrate (AZM), Clarithromycin (CTM), Doxycycline hyclate (DXC), Furosemide (FUS), Loratadine (LTD), Nitrofurantoin (NFT), Ondansetron hydrochloride dihydrate (ODS) from Tokyo Chemical Industry Co., Ltd. (Japan), Digoxin (DGX) from Alfa Aesar (U.S.A.), Ethosuximide (ESX), Metoclopramide hydrochloride (MCP), Propylthiouracil (PTU) from Toronto Research Chemicals, Inc. (Canada), Fluconazole (FCZ), Quinine hydrochloride (QNN) from Sigma-Aldrich Co. (U.S.A.), Isoniazid (ISZ) from LKT Laboratories, Inc. (U.S.A.), Metformin hydrochloride (MEF), Miconazole (MCZ), Paracetamol (PCM) from MP Biomedicals, LLC (U.S.A.) and Propranolol hydrochloride (PPL) from Matrix Scientific Inc. (Columbia).

Taste Sensor Measurement The taste sensor SA402B (Intelligent Sensor Technology Inc., Atsugi, Japan) was used to measure the membrane potential of the particular sensor against the reference electrode of 48 sample solutions in order to evaluate their bitterness. ${ }^{17-20,22)}$ The lipid/polymer membranes used as bitterness taste sensors in this study were as follows: for detecting basic bitterness, AC0 (hexadecanoic acid/dioctyl phenylphosphonate), AN0 (phosphoric acid di- $n$ decyl ester/dioctyl phenylphosphonate) and BT0 (phosphoric acid di- $n$-decyl ester/bis(1-butylpentyl) adipate, tributyl $O$ acetylcitrate); for detecting astringency, AE1 (tetradodecylammonium bromide/dioctyl phenylphosphonate); and for detecting acidic bitterness, $\mathrm{C} 00$ (tetradodecylammonium bromide/ 2-nitrophenyl octyl ether). ${ }^{19)}$

Each of the 48 APIs studied was dissolved in water to prepare solutions of $0.01,0.03$ and $0.1 \mathrm{mM}$ (Miconazole was dissolved in water to prepare solutions of $0.001,0.003$ and $0.01 \mathrm{mM}$ due to extremely low solubility.) for testing in the bitterness taste sensor. A semilogarithmic graph was plotted with the abscissa denoting compound concentration (mM; log scale) and the ordinate denoting sensor output $(\mathrm{mV})$. The slope of the plot was calculated and defined as CCDP. The CCDPs of the 48 APIs were analyzed by principal component analysis (PCA) using BellCurve for Excel ${ }^{\circledR}$ (Social Survey Research Information Co., Ltd.). We measured the membrane potential of the particular sensor against the reference electrode of three concentrations $(0.01,0.03$ and $0.1 \mathrm{mM})$ in quinine hydrochloride (standard substance for bitterness) solution at each time of the measurement, and performed reproducible confirmation every time.

Physicochemical Parameters Because it was thought that an electric charge and hydrophobicity participated in reactivity from a potentiometric principle of instrument using in this study, we selected parameters which participated in an electric charge and hydrophobicity. The physicochemical parameters except for $\log D$ of the 48 APIs were derived from the DRUGBANK database (https://www.drugbank.ca/). Log $D$ values ( $\mathrm{pH}$ 6.8) were derived from Marvinsketch (ChemAxon Ltd., Hungary). The 11 physicochemical parameters used in this study were as follows: molecular weight (MW), water solubility (WS), $\log P, \log D, \log S$ (predicted by ALOGPS), physiological charge, polar surface area (PSA), polarizability, hydrogen bond acceptor value, hydrogen bond donor value and number of aromatic bonds. 
Correlation between Physicochemical Properties and Taste Sensor Responses To determine the correlation coefficients by Fisher's exact tests, physicochemical properties were replaced by either 1 (high) or 0 (low), according to the method described in a previous article. ${ }^{26,34)}$ Thus, if the property of a compound was equal to or larger than that of QNN, the parameter was given the value 1; if the property was less than that of QNN, it was given the value 0. In the case of charge, if the absolute value of the property was equal to or larger than that of QNN, the parameter was given the value 1; if the absolute value of the property was less than that of QNN, it was given the value 0 . The slopes of the taste sensor dosedependent outputs (C00, AE1, AC0, AN0 and BT0) for each of the 48 APIs were also replaced by either 1 (response) or 0 (no response) as follows: if the slope of a compound was equal to or greater than that of QNN, the parameter was given the value 1; if the slope was less than that of QNN, it was given the value 0 .

Multiple regression analysis was performed to examine the correlation between CCDPs of AC0, AN0 and BT0 and physicochemical properties, with physicochemical properties as explanatory variables and CCDPs as target variables.

Molecular Modeling and Ligand Docking Simulations The hTAS2R14 model was built by submission of the amino acid sequence of hTAS2R14 obtained from National Center for Biotechnology Information (NCBI) to the I-TASSER server ${ }^{40)}$ in accordance with a previous report, ${ }^{37)}$ and the predicted structure of hTAS2R14 was then refined and minimized in energy using the Prime module in the Schrödinger suite (ver. 10.3). The generated structure of hTAS2R14 and the chemical structures of the studied drugs were prepared appropriately and docking simulations performed in accordance with previous reports. ${ }^{41)}$ Finally, the positions of the docked ligands were scored via the Glide module, and the docking structures were illustrated with molecular visualizing software PyMOL (Schrödinger).

Statistical Analysis BellCurve for Excel $^{\circledR}$ was used for statistical analysis using Fisher's exact tests and multiple regression analysis. Correlations between the slope of the taste sensor output values and the docking scores were evaluated using Pearson's test. The 5\% level of probability was considered significant.

Acknowledgments This work was supported by Grantsin-Aid for Scientific Research (C) from the Japan Society for Promotion of Science 16K08426 (to Takahiro Uchida) and 16K08425 (to Miyako Yoshida).

Conflict of Interest The authors declare no conflict of interest.

\section{References}

1) Thabet Y., Klingmann V., Breitkreutz J., J. Clin. Pharmacol., 58, 26-35 (2018)

2) Preis M., AAPS PharmSciTech, 16, 234-241 (2015).

3) Slavkova M., Breitkreutz J., Eur. J. Pharm. Sci., 75, 2-9 (2015).

4) Van Riet-Nales D. A., Kozarewicz P., Aylward B., de Vries R., Egberts T. C., Rademaker C. M., Schobben A. F., AAPS PharmSciTech, 18, 241-249 (2017)

5) Batchelor H. K., Fotaki N., Klein S., Adv. Drug Deliv. Rev., 73, 102-126 (2014).
6) Amin F., Khan S., Shah S. M. H., Rahim H., Hussain Z., Sohail M., Ullah R., Alsaid M. S., Shahat A. A., Drug Des. Devel. Ther., 12, 3855-3866 (2018)

7) Kendrick J. G., Ma K., Dezorzi P., Hamilton D., Can. J. Hosp. Pharm., 65, 196-201 (2012).

8) Mennella J. A., Beauchamp G. K., Clin. Ther., 30, 2120-2132 (2008).

9) Abraham J., Mathew F., Int. J. Pharm. Pharm. Sci., 6, 12-19 (2014).

10) Faisal W., Farag F., Abdellatif A. A. H., Abbas A., Curr. Drug Deliv., 15, 167-185 (2018).

11) Han C. S., Kim S., Oh D. W., Yoon J. Y., Park E. S., Rhee Y. S., Kim J. Y., Shin D. H., Kim D. W., Park C. W., Materials (Basel), 12, 1000 (2019)

12) Petrovick G. F., Breitkreutz J., Pein-Hackelbusch M., Int. J. Pharm., 506, 361-370 (2016).

13) Sharma S., Lewis S., Int. J. Pharm. Pharm. Sci., 2, 6-13 (2010).

14) Tan D. C. T., Ong J. J., Gokhale R., Heng P. W. S., Int. J. Pharm., 547, 385-394 (2018).

15) Meyerhof W., Batram C., Kuhn C., Brockhoff A., Chudoba E., Bufe B., Appendino G., Behrens M., Chem. Senses, 35, 157-170 (2010).

16) Di Pizio A., Niv M. Y., Bioorg. Med. Chem., 23, 4082-4091 (2015).

17) Haraguchi T., Miyazaki A., Yoshida M., Uchida T., J. Pharm. Pharmacol., 65, 980-987 (2013).

18) Ito M., Yoshida M., Kobayashi Y., Hiraoka M., Ikezaki H., Uchida T., Sensors and Materials, 23, 483-492 (2011).

19) Kobayashi Y., Habara M., Ikezazki H., Chen R., Naito Y., Toko K., Sensors (Basel), 10, 3411-3443 (2010).

20) Uchida T., Yoshida M., Hazekawa M., Haraguchi T., Furuno H., Teraoka M., Ikezaki H., J. Pharm. Pharmacol., 65, 1312-1320 (2013).

21) Wu X., Onitake H., Haraguchi T., Tahara Y., Yatabe R., Yoshida M., Uchida T., Ikezaki H., Toko K., Sens. Actuators B Chem., 235, 11-17 (2016).

22) Yoshida M., Haraguchi T., Uchida T., Chem. Pharm. Bull., 62, 1252-1258 (2014).

23) Yoshimatsu J., Toko K., Tahara Y., Ishida M., Habara M., Ikezaki H., Kojima H., Ikegami S., Yoshida M., Uchida T., Sensors (Basel), 20, 3455 (2020).

24) Preis M., Grother L., Axe P., Breitkreutz J., Int. J. Pharm., 491, 8-16 (2015).

25) Harada T., Sakurai M., Hondo S., Yasui M., Owaki T., Yakugaku Zasshi, 134, 325-331 (2014).

26) Haraguchi T., Okuno T., Nishikawa H., Kojima H., Ikegami S., Yoshida M., Habara M., Ikezaki H., Uchida T., Chem. Pharm. Bull., 67, 1271-1277 (2019).

27) Rudnitskaya A., Kirsanov D., Blinova Y., Legin E., Seleznev B. Clapham D., Ives R. S., Saunders K. A., Legin A., Anal. Chim. Acta, 770, 45-52 (2013).

28) Wesoly M., Kluk A., Sznitowska M., Ciosek P., Wroblewski W., Sensors (Basel), 16, 1353 (2016).

29) Born S., Levit A., Niv M. Y., Meyerhof W., Behrens M., J. Neurosci., 33, 201-213 (2013).

30) Roland W. S., van Buren L., Gruppen H., Driesse M., Gouka R. J., Smit G., Vincken J. P., J. Agric. Food Chem., 61, 10454-10466 (2013).

31) Ertl P., Rohde B., Selzer P., J. Med. Chem., 43, 3714-3717 (2000).

32) Clark D. E., J. Pharm. Sci., 88, 807-814 (1999).

33) Ghose A. K., Pritchett A., Crippen G. M., J. Comput. Chem., 9, 80-90 (1988)

34) Haraguchi T., Uchida T., Yoshida M., Kojima H., Habara M., Ikezaki H., Chem. Pharm. Bull., 66, 71-77 (2018).

35) Levit A., Nowak S., Peters M., Wiener A., Meyerhof W., Behrens M., Niv M. Y., FASEB J., 28, 1181-1197 (2014).

36) Nowak S., Di Pizio A., Levit A., Niv M. Y., Meyerhof W., Behrens M., Biochim. Biophys. Acta, Gen. Subj., 1862, 2162-2173 (2018).

37) Zhang Y., Wang X., Li X., Peng S., Wang S., Huang C. Z., Huang C. Z., Zhang Q., Li D., Jiang J., Ouyang Q., Zhang Y., Li S., Qiao 
Y., Sci. Rep., 7, 12174 (2017).

38) Sandal M., Behrens M., Brockhoff A., Musiani F., Giorgetti A., Carloni P., Meyerhof W., J. Chem. Theory Comput., 11, 4439-4449 (2015).

39) Karaman R., Nowak S., Di Pizio A., Kitaneh H., Abu-Jaish A., Meyerhof W., Niv M. Y., Behrens M., Chem. Biol. Drug Des., 88, 66-75 (2016).
40) Zhang J., Yang J., Jang R., Zhang Y., Structure, 23, 1538-1549 (2015)

41) Ōkuno T., Morimoto S., Nishikawa H., Haraguchi T., Kojima H., Tsujino H., Arisawa M., Yamashita T., Nishikawa J., Yoshida M., Habara M., Ikezaki H., Uchida T., Chem. Pharm. Bull., 68, 234-243 (2020). 